\title{
Two-band electronic metal and neighboring spin Bose-metal on a zigzag strip with longer-ranged repulsion
}

\author{
Hsin-Hua Lai and Olexei I. Motrunich \\ Department of Physics, California Institute of Technology, Pasadena, California 91125, USA
}

(Received 11 November 2009; published 5 January 2010)

\begin{abstract}
We consider an electronic model for realizing the spin Bose-metal (SBM) phase on a two-leg triangular strip — a spin liquid phase found by Sheng et al. [Phys. Rev. B 79, 205112 (2009)] in a spin-1/2 model with ring exchanges. The SBM can be viewed as a "C1S2" Mott insulator of electrons where the overall charge transporting mode is gapped out. We start from a two-band "C2S2" metal and consider extended repulsion motivated by recent $a b$ initio derivation of electronic model for $\kappa$-ET spin liquid material [K. Nakamura et al., J. Phys. Soc. Jpn. 78, 083710 (2009)]. Using weak coupling renormalization group analysis, we find that the extended interactions allow much wider $\mathrm{C} 2 \mathrm{~S} 2$ metallic phase than in the Hubbard model with on-site repulsion only. An eight-fermion umklapp term plays a crucial role in producing a Mott insulator but cannot be treated in weak coupling. We use bosonization to extend the analysis to intermediate coupling and study phases obtained out of the C2S2 metal upon increasing overall repulsion strength, finding that the SBM phase is a natural outcome for extended interactions.
\end{abstract}

DOI: 10.1103/PhysRevB.81.045105

PACS number(s): 71.10.Hf, 71.10.Pm

\section{INTRODUCTION}

There has been much recent interest in gapless spin liquids stimulated by the appearance of several experimental candidates, including two-dimensional (2D) triangular lattice based organic compounds ${ }^{1-5} \quad \kappa-(\mathrm{ET})_{2} \mathrm{Cu}_{2}(\mathrm{CN})_{3}$ and $\mathrm{EtMe}_{3} \mathrm{Sb}\left[\mathrm{Pd}(\mathrm{dmit})_{2}\right]_{2}$ and three-dimensional (3D) hyperkagome material ${ }^{6} \mathrm{Na}_{4} \mathrm{Ir}_{3} \mathrm{O}_{8}$. One line of theoretical ideas considers states with a Fermi surface of fermionic spinons. ${ }^{7-10}$ For the 2D spin liquids, such a state arises as a good variational wave function ${ }^{7}$ for an appropriate spin model with ring exchanges; it is also an appealing candidate for the Hubbard model near the Mott transition. ${ }^{8,11,12}$ Theoretical description of such states leads to a U(1) gauge theory (see Ref. 13 for a review).

Variational studies are not sufficient to prove that a given state is realized and the gauge theory is not fully reliable in 2D. Driven by the need for a controlled theoretical access to such phases, Ref. 14 considered the Heisenberg plus ring exchanges model on a two-leg triangular strip (so-called zigzag chain). ${ }^{15,16}$ Using numerical density matrix renormalization group (DMRG), variational Monte Carlo (VMC), and analytical bosonization treatment, Ref. 14 found a ladder descendant of the 2D spin liquid in a broad range of parameters and dubbed this phase "spin Bose metal" (SBM). The name refers to metal-like itinerancy present in the spin degrees of freedom, while there is no electric transport to speak of in the spin-only model, which is bosonic model microscopically.

A low-energy field theory ${ }^{14}$ for the zigzag SBM phase can be obtained by employing bosonization to analyze the spinon-gauge theory (the slave particle approach also underlies the VMC trial states). An alternative derivation of the SBM theory is to consider an interacting model of electrons hopping on the zigzag chain and to drive a transition from a two-band metal to a particular Mott insulator. Specifically, let us start in the metallic phase with two gapless charge modes and two gapless spin modes-so-called "C2S2" metal. We can imagine gapping out just the overall charge mode to obtain a "C1S2" Mott insulator with one gapless "charge" mode and two gapless spin modes, where the former represents local current loop fluctuations and does not transport charge along the chain. This is precisely the SBM phase. If one thinks of a spin-only description of this Mott insulator, the gapless charge mode can be interpreted as spin singlet chirality mode. Ref. 14 also identified a valid umklapp term that can drive the electron system to the C1S2 phase.

In this paper, we focus on realizing such scenario for the SBM in explicit and realistic electronic models. Hubbard model on the zigzag chain $\left(t_{1}-t_{2}-U\right.$ chain) has received much attention. ${ }^{17-22}$ For free electrons, the two-band metal appears for $t_{2} / t_{1}>0.5$. However, in the case of Hubbard interaction, weak coupling approach ${ }^{18,19}$ finds that this phase is stable only over a narrow range $t_{2} / t_{1} \in[0.5,0.57]$, while a spin gap opens up for larger $t_{2} / t_{1}$. The umklapp that can drive a transition to a Mott insulator requires eight fermions and is strongly irrelevant at weak coupling. Prior work ${ }^{17,18,23}$ focused on the spin-gapped metal and eventual spin-gapped insulator for strong interaction, while the C1S2 spin liquid phase was not anticipated.

There have also been numerical DMRG studies of the Hubbard model. ${ }^{19-22}$ The focus has been on the prominent spin-gapped phases and, in particular, on the insulator that is continuously connected to the dimerized phase in the $J_{1}-J_{2}$ Heisenberg model, which is appropriate in the strong interaction limit $U \gg t_{1}, t_{2}$. The C2S2 metallic phase and possibility of nearby spin liquid on the Mott insulator side in the Hubbard model have not been explored. We hope our work will motivate more studies of this interesting possibility in the Hubbard model with intermediate $U$ close to the C2S2 metal.

Since the C2S2 metallic phase is quite narrow in the Hubbard model, we would like to first widen the $\mathrm{C} 2 \mathrm{~S} 2$ region. To this end, we explore an electronic model with extended repulsive interactions. ${ }^{24}$ Such interactions tend to suppress instabilities in the electronic system, similar to how longranged Coulomb repulsion suppresses pairing in metals. 
They are also more realistic than the on-site Hubbard, particularly for materials undergoing a metal-insulator transition where there is no conduction band screening on the insulator side. Thus, recent $a b$ initio model construction for the $\kappa-(\mathrm{ET})_{2} \mathrm{Cu}_{2}(\mathrm{CN})_{3}$ material found significant extended interactions in the corresponding electronic model on the halffilled triangular lattice. ${ }^{25,26}$

Applying weak coupling renormalization group (RG) approach to the zigzag ladder system, ${ }^{17,18,23,27}$ we indeed find that extended interactions open a much wider window of the C2S2 metal phase. Building on this, we then use bosonization approach to explore a transition to a Mott insulator upon increasing the overall repulsion strength. We find that such longer-ranged interactions can drive the system into the C1S2 spin liquid Mott insulator rather than a spin-gapped insulator. This bodes well for finding spin liquid phases in more realistic electronic models for materials near the metalinsulator transition.

The paper is organized as follows. In Sec. II, we set up the weak coupling RG (Refs. 18, 23, and 27) and open a much wider window of the metallic $\mathrm{C} 2 \mathrm{~S} 2$ phase by introducing realistically motivated longer-ranged repulsion. In Sec. III, we use bosonization to extend the analysis to intermediate coupling. We gradually increase the overall repulsion strength and determine thresholds for a Mott transition driven by the eight-fermion umklapp term and also for spin gap instabilities, thus mapping out phases neighboring the C2S2 metal. In Sec. IV, we summarize our results and conclude with some discussion.

\section{WEAK COUPLING ANALYSIS OF $t_{1}-t_{2}$ MODEL WITH EXTENDED REPULSION: STABILIZING C2S2 METAL}

\section{A. Setup for two-band electron system}

We consider half-filled electronic $t_{1}-t_{2}$ model with extended interaction described by the Hamiltonian $H=H_{0}+H_{V}$, with

$$
H_{0}=-\sum_{x, \alpha}\left[t_{1} c_{\alpha}^{\dagger}(x) c_{\alpha}(x+1)+t_{2} c_{\alpha}^{\dagger}(x) c_{\alpha}(x+2)+\text { H.c. }\right],
$$

$$
H_{V}=\frac{1}{2} \sum_{x, x^{\prime}} V\left(x-x^{\prime}\right) n(x) n\left(x^{\prime}\right) .
$$

Here $c\left(c^{\dagger}\right)$ is fermion annihilation (creation) operator, $x$ is a site label on the one-dimensional (1D) chain, and $\alpha=\uparrow, \downarrow$ is a spin index; $n(x) \equiv \Sigma_{\alpha} c_{\alpha}^{\dagger}(x) c_{\alpha}(x)$ is electron number on the site.

In weak coupling, the kinetic energy Eq. (1) gives free particle dispersion

$$
\epsilon(k)=-2 t_{1} \cos (k)-2 t_{2} \cos (2 k) .
$$

For $t_{2} / t_{1}>0.5$, there are two sets of Fermi points at wavevectors $\pm k_{F 1}$ and $\pm k_{F 2}$ as shown in Fig. 1. We adopt the same conventions as in Ref. 14. Fermions near $k_{F 1}$ and $k_{F 2}$ are moving to the right, and the corresponding group

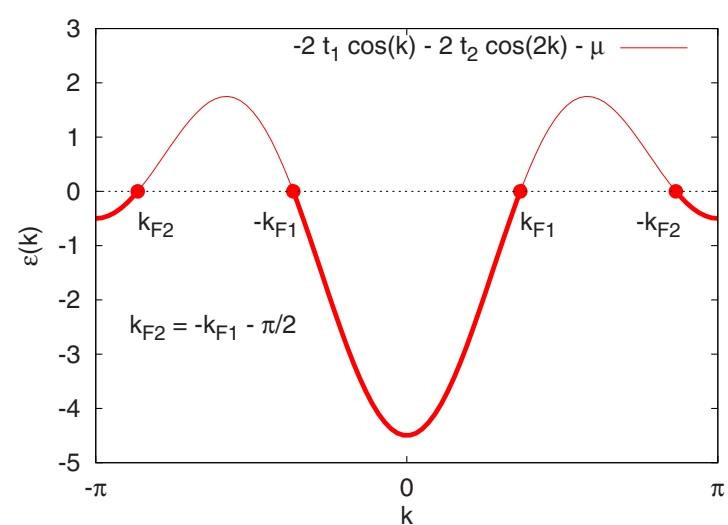

FIG. 1. (Color online) Electron band for $t_{2}>0.5 t_{1}$ has two occupied Fermi sea segments. This is free fermion C2S2 metal.

velocities are $v_{1}, v_{2}>0$. Electrons are at half-filling, which implies $k_{F 1}+k_{F 2}=-\pi / 2 \bmod 2 \pi$ for the choices as in Fig. 1 .

The electron operators are expanded in terms of continuum fields,

$$
c_{\alpha}(x)=\sum_{P, a} e^{i P k_{F a} x} c_{P a \alpha},
$$

with $P=R / L=+/-$ denoting the right and left movers and $a=1,2$ denoting the two Fermi seas.

Four-fermion interactions can be conveniently expressed in terms of chiral currents, ${ }^{14,23,27}$

$$
\begin{gathered}
J_{P a b}=\sum_{\alpha} c_{P a \alpha}^{\dagger} c_{P b \alpha}, \\
\vec{J}_{P a b}=\sum_{\alpha, \beta} c_{P a \alpha}^{\dagger} \frac{\vec{\sigma}_{\alpha \beta}}{2} c_{P b \beta} .
\end{gathered}
$$

Most general four-fermion interactions can be written as

$$
\begin{gathered}
\mathcal{H}_{R L}^{\rho}=\sum_{a, b}\left(w_{a b}^{\rho} J_{R a b} J_{L a b}+\lambda_{a b}^{\rho} J_{R a a} J_{L b b}\right), \\
\mathcal{H}_{R L}^{\sigma}=-\sum_{a, b}\left(w_{a b}^{\sigma} \vec{J}_{R a b} \cdot \vec{J}_{L a b}+\lambda_{a b}^{\sigma} \vec{J}_{R a a} \cdot \vec{J}_{L b b}\right), \\
\mathcal{H}_{\text {chiral }}^{\rho}=\frac{1}{2} \sum_{a} C_{a a}^{\rho}\left(J_{R a a} J_{R a a}+J_{L a a} J_{L a a}\right) \\
+C_{12}^{\rho}\left(J_{R 11} J_{R 22}+J_{L 11} J_{L 22}\right), \\
\mathcal{H}_{\text {chiral }}^{\sigma}=-\frac{1}{2} \sum_{a} C_{a a}^{\sigma}\left(\vec{J}_{R a a} \cdot \vec{J}_{R a a}+\vec{J}_{L a a} \cdot \vec{J}_{L a a}\right) \\
-C_{12}^{\sigma}\left(\vec{J}_{R 11} \cdot \vec{J}_{R 22}+\vec{J}_{L 11} \cdot \vec{J}_{L 22}\right) .
\end{gathered}
$$

Here $\mathcal{H}_{R L}$ are terms that connect right and left movers, while $\mathcal{H}_{\text {chiral }}$ are chiral terms with all fermions moving in the same direction.

Consider the couplings in $\mathcal{H}_{R L}$. We have $w_{11}=w_{22}=0$ (convention), $w_{12}=w_{21}$ (from Hermiticity), and $\lambda_{12}=\lambda_{21}$ (from $R \leftrightarrow L$ symmetry). Thus there are 8 independent couplings: $w_{12}^{\rho / \sigma}, \lambda_{11}^{\rho / \sigma}, \lambda_{22}^{\rho / \sigma}$, and $\lambda_{12}^{\rho / \sigma}$. Note that there are no four- 
fermion umklapp terms in our two-band system.

In the specific lattice model, we expand the interactions [Eq. (2)] in terms of the continuum fields and find "bare" values of the couplings,

$$
\begin{gathered}
\lambda_{11}^{\rho}=V_{Q=0}-\frac{V_{2 k_{F 1}}}{2}, \\
\lambda_{22}^{\rho}=V_{Q=0}-\frac{V_{2 k_{F 2}}}{2}, \\
\lambda_{12}^{\rho}=V_{Q=0}-\frac{V_{\pi / 2}}{2}, \\
\lambda_{11}^{\sigma}=2 V_{2 k_{F 1}}, \\
\lambda_{22}^{\sigma}=2 V_{2 k_{F 2}}, \\
\lambda_{12}^{\sigma}=2 V_{\pi / 2}, \\
C_{12}^{\rho}=V_{Q=0}-\frac{V_{k_{F 1}-k_{F 2}}}{2}, \\
C_{12}^{\sigma}=V_{k_{F 1}-k_{F 2}}-\frac{V_{\pi / 2}}{2}, \\
w_{12}^{\sigma}=2 V_{k_{F 1}}, C_{22}^{\rho}=V_{Q=0}-\frac{U}{2}, \\
{ }_{12}=2 U,
\end{gathered}
$$

Here $\quad V_{Q} \equiv \sum_{x^{\prime}=-\infty}^{\infty} V\left(x-x^{\prime}\right) e^{i Q\left(x-x^{\prime}\right)}=V_{-Q} \quad$ since $\quad V\left(x-x^{\prime}\right)$ $=V\left(x^{\prime}-x\right)$. We have also used explicitly $k_{F 1}+k_{F 2}=-\pi / 2$.

The terms $\mathcal{H}_{\text {chiral }}$ renormalize "velocities" of various modes. In the weak coupling RG analysis, they only generate higher-order contributions and are therefore not important. The RG equations below contain only couplings from $\mathcal{H}_{R L}$. On the other hand, the chiral interactions are important in the intermediate coupling analysis to be done in Sec. III, which is why we have listed their values as well. The on-site coupling $U \equiv V\left(x-x^{\prime}=0\right)$ appears explicitly in $C_{11}^{\rho / \sigma}$ and $C_{22}^{\rho / \sigma}$ because of our more careful treatment of the on-site interaction, which we first write as $U n_{\uparrow}(x) n_{\downarrow}(x)$ and then insert the continuum fields (and bosonize in Sec. III).

\section{B. Weak coupling renormalization group}

The RG equations in the two-band system are a, $^{18,27}$

$$
\dot{\lambda}_{11}^{\rho}=-\frac{1}{2 \pi v_{2}}\left[\left(w_{12}^{\rho}\right)^{2}+\frac{3}{16}\left(w_{12}^{\sigma}\right)^{2}\right] \text {, }
$$

$$
\begin{gathered}
\dot{\lambda}_{22}^{\rho}=-\frac{1}{2 \pi v_{1}}\left[\left(w_{12}^{\rho}\right)^{2}+\frac{3}{16}\left(w_{12}^{\sigma}\right)^{2}\right], \\
\dot{\lambda}_{12}^{\rho}=\frac{1}{\pi\left(v_{1}+v_{2}\right)}\left[\left(w_{12}^{\rho}\right)^{2}+\frac{3}{16}\left(w_{12}^{\sigma}\right)^{2}\right], \\
\dot{\lambda}_{11}^{\sigma}=-\frac{1}{2 \pi v_{1}}\left(\lambda_{11}^{\sigma}\right)^{2}-\frac{1}{4 \pi v_{2}}\left[\left(w_{12}^{\sigma}\right)^{2}+4 w_{12}^{\rho} w_{12}^{\sigma}\right], \\
\dot{\lambda}_{22}^{\sigma}=-\frac{1}{2 \pi v_{2}}\left(\lambda_{22}^{\sigma}\right)^{2}-\frac{1}{4 \pi v_{1}}\left[\left(w_{12}^{\sigma}\right)^{2}+4 w_{12}^{\rho} w_{12}^{\sigma}\right], \\
\dot{\lambda}_{12}^{\sigma}=-\frac{1}{\pi\left(v_{1}+v_{2}\right)}\left\{\left(\lambda_{12}^{\sigma}\right)^{2}+\frac{\left(w_{12}^{\sigma}\right)^{2}-4 w_{12}^{\rho} w_{12}^{\sigma}}{2}\right\}, \\
\dot{w}_{12}^{\sigma}=-\Lambda_{12}^{\sigma} w_{12}^{\rho}-\left(\Lambda^{\rho}+\frac{\Lambda^{\sigma}}{2}+\frac{2 \lambda_{12}^{\sigma}}{\pi\left(v_{1}+v_{2}\right)}\right) w_{12}^{\sigma} .
\end{gathered}
$$

Here $\dot{O} \equiv \partial O / \partial \ell$, where $\ell$ is logarithm of the length scale. We have also defined

$$
\Lambda^{\rho / \sigma}=\frac{\lambda_{11}^{\rho / \sigma}}{2 \pi v_{1}}+\frac{\lambda_{22}^{\rho / \sigma}}{2 \pi v_{2}}-\frac{2 \lambda_{12}^{\rho / \sigma}}{\pi\left(v_{1}+v_{2}\right)} .
$$

Details of our system enter through the band velocities $v_{1}$, $v_{2}$, and the initial conditions [Eqs. (11)-(18)].

\section{Fixed point for stable C2S2 phase}

We are primarily interested in the stability of the twoband metallic phase with two gapless charge and two gapless spin modes-C2S2 in the notation of Ref. 23. In the RG, this phase is characterized as having no divergent couplings. Before proceeding with detailed numerical studies of the flow Eqs. (23)-(30), we can describe such stable C2S2 fixed point qualitatively: the charge sector couplings reach some fixed values, $\lambda_{11}^{\rho^{*}}, \lambda_{22}^{\rho^{*}}$, and $\lambda_{12}^{\rho^{*}}$, and are strictly marginal; they also need to satisfy $\Lambda^{\rho^{*}}>0$ (see below). The spin sector couplings approach zero from positive values, $\lambda_{11}^{\sigma^{*}}=\lambda_{22}^{\sigma^{*}}=\lambda_{12}^{\sigma^{*}}$ $=0^{+}$, and are marginally irrelevant. Finally, the "charge-spin" couplings $w_{12}$ go to zero, $w_{12}^{\rho *}=w_{12}^{\sigma *}=0$, and are irrelevant, which is ensured by the condition $\Lambda^{\rho^{*}}>0$. Indeed, consider small deviations of comparable magnitudes for all couplings and allowing only positive $\lambda_{a b}^{\sigma}$. Since we have finite $\Lambda^{\rho^{*}}$ $>0$, first the $w_{12}^{\rho / \sigma}$ will renormalize quickly to zero, without affecting significantly the other couplings. Then the $\lambda_{a b}^{\sigma}$ will renormalize to zero via slow marginal flows.

\section{Numerical studies of the flows}

We can solve the RG equations numerically for given initial conditions and check whether the couplings flow into the domain of attraction of the $\mathrm{C} 2 \mathrm{~S} 2$ fixed point or not. We 


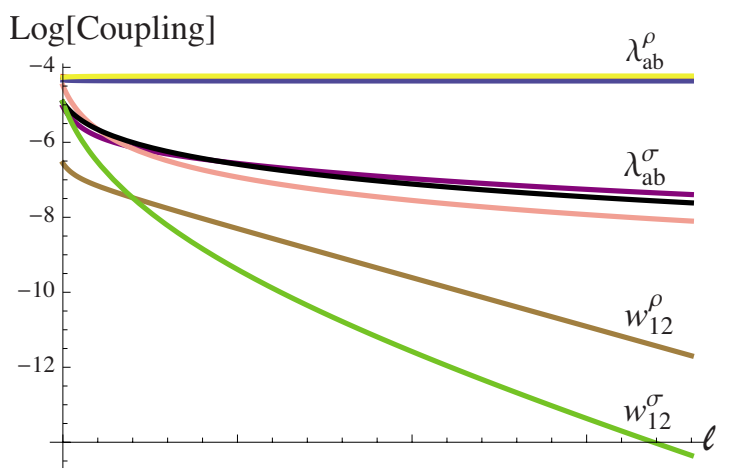

FIG. 2. (Color online) Example of RG flows in the C2S2 phase. The model potential is Eq. (32) with $\kappa=1 / 2, \gamma=2 / 5$; the band parameter is $t_{2} / t_{1}=0.9$. We choose logarithm of the couplings to be the $y$ axis and RG "time" $\ell$ to be the $x$-axis. We see that $w_{12}^{\rho / \sigma}$ flow toward 0 rapidly (irrelevant couplings); $\lambda_{a b}^{\rho}$ saturate very fast (strictly marginal couplings); while $\lambda_{a b}^{\sigma}$ flow to 0 slowly (marginally irrelevant). More generally, if we fix these $\kappa$ and $\gamma$ values, for $t_{2} / t_{1}<0.99$ the flows are similar to those shown here and the phase is $\mathrm{C} 2 \mathrm{~S} 2$.

use Mathematica to solve the flows up to long "time" $\ell$ when the ultimate trends become apparent.

If the couplings always remain of the same order as their initial values or approach zero, we say the couplings are marginal or irrelevant and identify this as the $\mathrm{C} 2 \mathrm{~S} 2$ phase. The eventual trends here were discussed in Sec. II C

On the other hand, if the magnitudes of some couplings grow significantly compared to the initial values, we say that the couplings are relevant and the $\mathrm{C} 2 \mathrm{~S} 2$ phase is destroyed. Thus, if either $\lambda_{11}^{\sigma}$ or $\lambda_{22}^{\sigma}$ coupling becomes negative while $w_{12}^{\sigma}$ and $w_{12}^{\rho}$ remain of the same sign, this $\lambda^{\sigma}$ then runs away to large negative values and also induces the other couplings to diverge. Bosonizing the four-fermion interactions ${ }^{14,23,27}$ (cf. Sec. III), we can see that two spin modes and one charge mode become gapped and we obtain so-called "C1SO" phase. The overall charge propagation mode remains gapless and the system is conducting. Note that we do not distinguish which coupling diverges faster in the formal flow [Eqs. (23)-(30)]. As discussed in Ref. 23, in the $U \rightarrow 0+$ limit one can separate a so-called $\mathrm{C} 2 \mathrm{~S} 1$ case where one of the spin couplings diverges qualitatively faster (but all couplings still diverge at the same $\ell$ ). We do not make such subtle distinction and call any runaway flow situation as $\mathrm{C} 1 \mathrm{SO}$-all we want to know is that the two-band metal $\mathrm{C} 2 \mathrm{~S} 2$ became unstable.

The RG flows are qualitatively similar for different points in the same phase, so we only show one representative picture for each case. Figure 2 shows the flows in the C2S2 phase. The scale parameter $\ell$ is the $x$ axis, while logarithm of the couplings is the $y$ axis. In this way, we clearly see that the couplings separate into three groups, which is well explained by the C2S2 fixed point in Sec. II C: the $w_{12}^{\rho / \sigma}$ flow to 0 exponentially rapidly, the $\lambda_{a b}^{\sigma}$ flow to 0 marginally slowly, while the $\lambda_{a b}^{\rho}$ saturate.

Figure 3 illustrates the flows in the C1S0 phase. Here we use real values of the coupling as the $y$ axis and only show selected couplings, $\lambda_{11}^{\sigma}, \lambda_{22}^{\sigma}, w_{12}^{\rho}$, and $w_{12}^{\sigma}$. We clearly see that

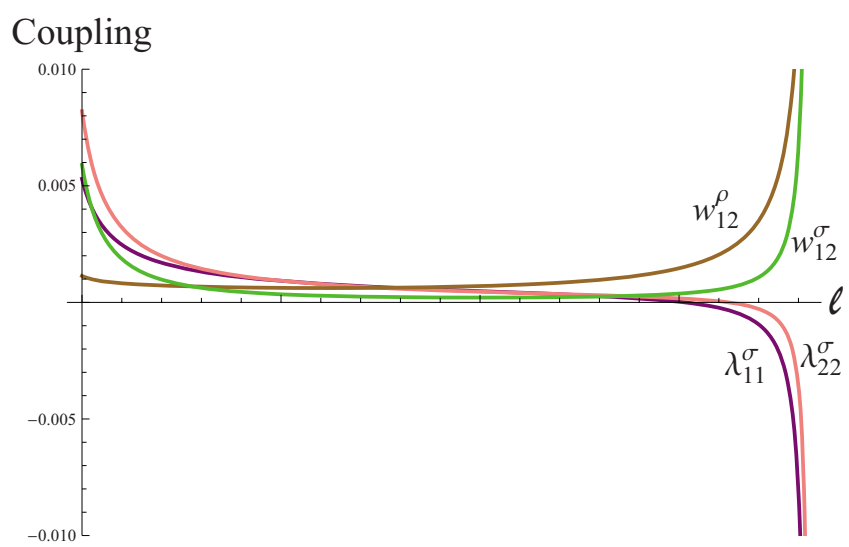

FIG. 3. (Color online) Example of RG flows of selected couplings in the C1S0 phase. The model is the same as in Fig. 2, but with $t_{2} / t_{1}=1.05$. We see that the selected couplings diverge after some time. For example, once the $\lambda_{11}^{\sigma}$ and $\lambda_{22}^{\sigma}$ become negative while $w_{12}^{\rho}$ and $w_{12}^{\sigma}$ remain positive, the RG Eqs. (23)-(30) drive the $\lambda_{11}^{\sigma}$ and $\lambda_{22}^{\sigma}$ to $-\infty$ and in turn $w_{12}^{\rho}$ and $w_{12}^{\sigma}$ to $+\infty$, and then all couplings diverge. More generally, if we fix $\gamma=2 / 5$, for $t_{2} / t_{1}$ $>0.99$ the flows are similar to those shown here and we call this C1S0 phase. Varying $\gamma$, we obtain the phase diagram Fig. 4.

these couplings diverge (and so do the other couplings not shown in the figure).

\section{E. Examples of phase diagrams with C2S2 metal stabilized by extended interactions}

For illustration in our paper, we consider the following interaction potential,

$$
V\left(x-x^{\prime}\right)=\left\{\begin{array}{cc}
U, & \left|x-x^{\prime}\right|=0 \\
\kappa U e^{-\gamma\left|x-x^{\prime}\right|}, & \left|x-x^{\prime}\right| \geq 1
\end{array}\right\} .
$$

Here $U$ is the overall energy scale and also the on-site repulsion. The relative magnitude of the extended repulsion is set by some factor $\kappa<1$. Beyond one lattice spacing, the potential decreases exponentially with decay rate $\gamma$. For $\gamma \rightarrow \infty$ we obtain the Hubbard model with on-site interaction only, while for small $\gamma$ the interaction extends over many lattice sites.

We also consider the above potential but truncated at the fourth neighbor. This tests robustness of our conclusions to modifications where the interactions have finite but still somewhat extended range, as may be preferable in numerical studies of such electronic models.

\section{Weak coupling phase diagram for potential [Eq. (32)]}

The extended repulsion, Eq. (32), is in Fourier space,

$$
V_{Q}=U\left[1-\kappa+\frac{\kappa \sinh (\gamma)}{\cosh (\gamma)-\cos (Q)}\right] .
$$

For given model parameters, we use Eqs. (11)-(18) to set initial conditions. We follow the RG flows and identify the phases as described above, thus mapping out the "weak coupling phase diagram." Here and in the rest of the paper, we take $\kappa=0.5$. This is loosely motivated by the recent $a b$ initio 


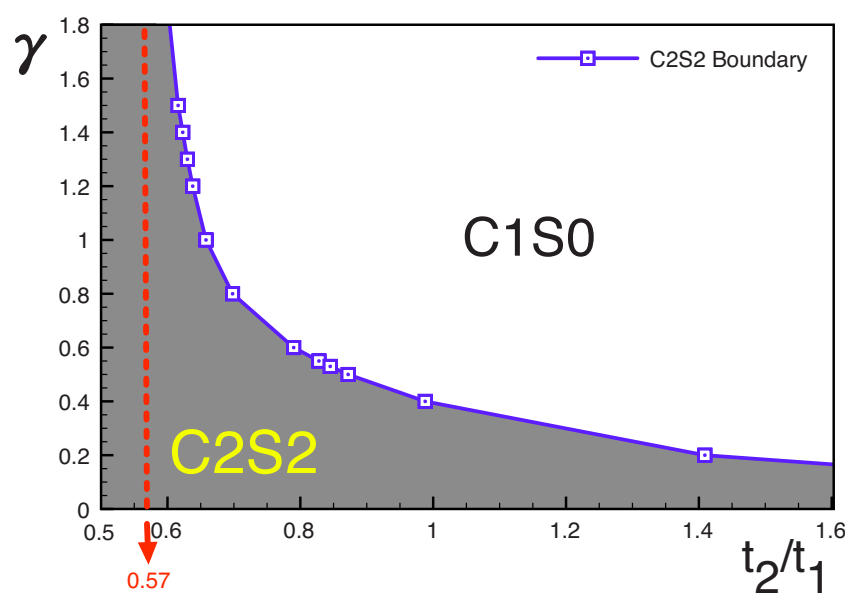

FIG. 4. (Color online) Stabilization of the C2S2 metal by extended interactions. The model potential is Eq. (32) with $\kappa=0.5$. The noninteracting problem has one band for $t_{2} / t_{1}<0.5$ and two bands for $t_{2} / t_{1}>0.5$, cf. Figure 1, and we focus on the latter region. The limit $\gamma \rightarrow \infty$ corresponds to the Hubbard model with on-site repulsion only, and the $\mathrm{C} 2 \mathrm{~S} 2$ phase is stable only over a narrow window $t_{2} / t_{1} \in[0.5 \cdots 0.57]$ (Refs. 18 and 23). The C2S2 region becomes progressively wider as we increase the interaction range $1 / \gamma$.

calculation $^{25}$ for the $\kappa$ - $(\mathrm{ET})_{2} \mathrm{Cu}_{2}(\mathrm{CN})_{3}$ which gives the ratio of the nearest-neighbor repulsion $V_{1} \equiv V\left(\left|x-x^{\prime}\right|=1\right)$ to the on-site Hubbard $U$ as $V_{1} / U \simeq 0.43$, while in our model $V_{1} / U=\kappa e^{-\gamma}$. The corresponding phase diagram showing stable C2S2 region is in Fig. 4.

We see that the $\mathrm{C} 2 \mathrm{~S} 2$ region becomes wider upon increasing the interaction range $1 / \gamma$. We can understand this qualitatively as follows. For fixed band parameters, when $\gamma \rightarrow 0$ the values of $V_{Q}$ for all nonzero $Q$ approach $U(1-\kappa)$, while $V_{Q=0} \simeq 2 \kappa U / \gamma$ continues to increase. The corresponding contribution to $\Lambda^{\rho}$ is

$$
\delta \Lambda^{\rho}=\frac{V_{Q=0}}{2 \pi}\left[\frac{1}{v_{1}}+\frac{1}{v_{2}}-\frac{4}{v_{1}+v_{2}}\right]=\frac{V_{Q=0}}{2 \pi}\left[\frac{\left(v_{1}-v_{2}\right)^{2}}{v_{1} v_{2}\left(v_{1}+v_{2}\right)}\right],
$$

which is positive for any $v_{1} \neq v_{2}$ and grows with increasing $V_{Q=0}$. Note also from Eqs. (11)-(18) that the $V_{Q=0}$ enters only in the $\lambda_{a b}^{\rho}$ couplings. Large bare value of $\Lambda^{\rho}$ makes the $w_{12}^{\rho / \sigma}$ flows strongly irrelevant. Their effect on the $\lambda_{a b}^{\sigma}$ flows is rapidly decreasing and expires. The $\lambda_{a b}^{\sigma}$ couplings start repulsive and stay so and eventually flow to zero via marginal flows. This argument is strictly true in the small $\gamma$ limit, while for finite $\gamma$ the interplay of different flows is more complex and requires numerical study as done in Fig. 4.

\section{Weak coupling phase diagram for potential [Eq. (32)] truncated at the fourth neighbor}

Here, we truncate the interaction at the fourth neighbor, so the Fourier transform is,

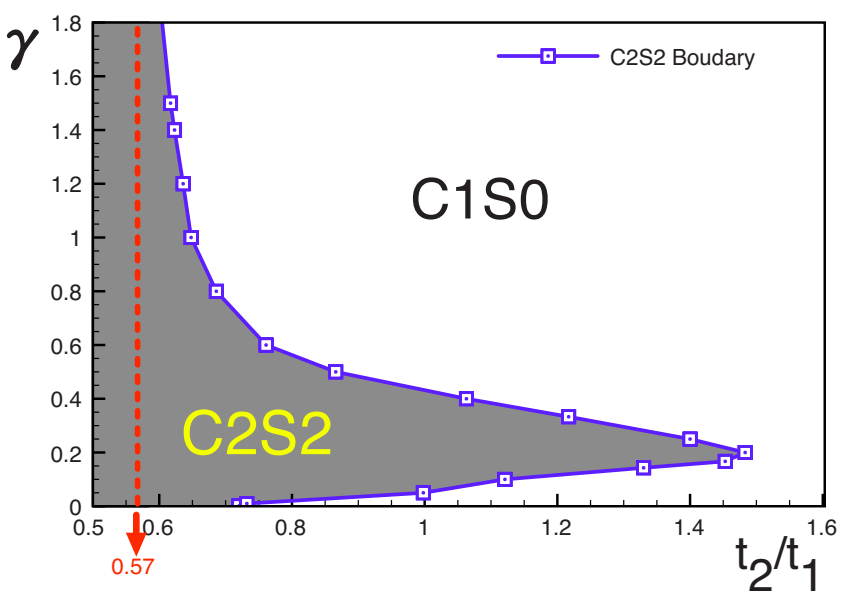

FIG. 5. (Color online) Same as Fig. 4 but for the potential Eq. (32) truncated at the fourth neighbor.

$$
V_{Q}=U\left[1+2 \kappa \sum_{n=1}^{4} e^{-n \gamma} \cos (n Q)\right] .
$$

The phase diagram in the weak coupling $\mathrm{RG}$ approach is shown in Fig. 5. We see that unlike the case without the truncation, the C1S0 phase opens again as $\gamma \rightarrow 0$. Since we only include up to the fourth neighbor interaction, $V_{Q=0}$ does not dominate over $V_{Q \neq 0}$ even in the $\gamma \rightarrow 0$ limit. For $\kappa=0.5$ and $\gamma=0$, there is significant structure in $V_{Q}$ including sign changes as a function of $Q$, which can make bare spin couplings $\lambda_{a a}^{\sigma} \sim V_{2 k_{F a}}$ to be marginally relevant. Nevertheless, for intermediate $\gamma$ there is still a wide window of the C2S2 phase.

\section{WEAK TO INTERMEDIATE COUPLING: PHASES OUT OF C2S2 UPON INCREASING INTERACTION}

\section{A. Harmonic description of the C2S2 phase}

Let us begin with a harmonic description of the C2S2 metal. Technical steps and many details of the bosonization essentially follow Ref. 14 and references therein. We write

$$
c_{P a \alpha}=\eta_{a \alpha} e^{i\left(\varphi_{a \alpha}+P \theta_{a \alpha}\right)},
$$

where $\varphi$ and $\theta$ are canonically conjugate boson fields and $\eta$ are Klein factors.

We define "charge" and "spin" boson fields,

$$
\theta_{a \rho / \sigma}=\frac{1}{\sqrt{2}}\left(\theta_{a \uparrow} \pm \theta_{a \downarrow}\right),
$$

and "even" and "odd" flavor combinations,

$$
\theta_{\mu \pm}=\frac{1}{\sqrt{2}}\left(\theta_{1 \mu} \pm \theta_{2 \mu}\right),
$$

with $\mu=\rho, \sigma$. Similar definitions hold for the $\varphi$ fields.

We can now bosonize all four-fermion interactions [Eqs. (7)-(10)]. First consider the spin sector. The $C_{a b}^{\sigma}$ terms give velocity renormalizations, while the $\lambda_{a b}^{\sigma}$ terms are written out in Sec. IVA of Ref. 14 and are not repeated here. We assume 
that the $\lambda_{a b}^{\sigma}$ are marginally irrelevant in the C2S2 phase. The fixed-point Lagrangian has effectively decoupled boson fields $\theta_{1 \sigma}$ and $\theta_{2 \sigma}$ with Luttinger parameters $g_{1 \sigma}=g_{2 \sigma}=1$, dictated by $\mathrm{SU}(2)$ spin rotation invariance.

The Lagrangian in the charge sector is

$$
\mathcal{L}^{\rho}=\frac{1}{2 \pi}\left[\partial_{x} \boldsymbol{\Theta}^{T} \cdot \mathbf{A} \cdot \partial_{x} \boldsymbol{\Theta}+\partial_{x} \boldsymbol{\Phi}^{T} \cdot \mathbf{B} \cdot \partial_{x} \boldsymbol{\Phi}\right]+\frac{i}{\pi} \partial_{x} \boldsymbol{\Theta}^{T} \cdot \partial_{\tau} \boldsymbol{\Phi},
$$

where we defined $\boldsymbol{\Theta}^{T}=\left(\theta_{\rho+}, \theta_{\rho-}\right)$ and $\boldsymbol{\Phi}^{T}=\left(\varphi_{\rho+}, \varphi_{\rho-}\right)$. Matrix elements of $\mathbf{A}$ and $\mathbf{B}$ are

$$
\begin{aligned}
& A_{11}=\bar{v}+\frac{\lambda_{11}^{\rho}+\lambda_{22}^{\rho}+2 \lambda_{12}^{\rho}}{2 \pi}+\frac{C_{11}^{\rho}+C_{22}^{\rho}+2 C_{12}^{\rho}}{2 \pi}=\bar{v}+\frac{4 V_{Q=0}}{\pi} \\
& -\frac{V_{2 k_{F 1}}}{4 \pi}-\frac{V_{2 k_{F 2}}}{4 \pi}-\frac{V_{\pi / 2}}{2 \pi}-\frac{V_{k_{F 1}-k_{F 2}}}{2 \pi}-\frac{U}{2 \pi}, \\
& A_{22}=\bar{v}+\frac{\lambda_{11}^{\rho}+\lambda_{22}^{\rho}-2 \lambda_{12}^{\rho}}{2 \pi}+\frac{C_{11}^{\rho}+C_{22}^{\rho}-2 C_{12}^{\rho}}{2 \pi}=\bar{v}-\frac{V_{2 k_{F 1}}}{4 \pi} \\
& -\frac{V_{2 k_{F 2}}}{4 \pi}+\frac{V_{\pi / 2}}{2 \pi}+\frac{V_{k_{F 1}-k_{F 2}}}{2 \pi}-\frac{U}{2 \pi} \\
& A_{12}=A_{21}=v_{r}+\frac{\lambda_{11}^{\rho}-\lambda_{22}^{\rho}}{2 \pi}+\frac{C_{11}^{\rho}-C_{22}^{\rho}}{2 \pi}=v_{r}-\frac{V_{2 k_{F 1}}}{4 \pi}+\frac{V_{2 k_{F 2}}}{4 \pi}, \\
& B_{11}=\bar{v}-\frac{\lambda_{11}^{\rho}+\lambda_{22}^{\rho}+2 \lambda_{12}^{\rho}}{2 \pi}+\frac{C_{11}^{\rho}+C_{22}^{\rho}+2 C_{12}^{\rho}}{2 \pi}=\bar{v}+\frac{V_{2 k_{F 1}}}{4 \pi} \\
& +\frac{V_{2 k_{F 2}}}{4 \pi}+\frac{V_{\pi / 2}}{2 \pi}-\frac{V_{k_{F 1}-k_{F 2}}}{2 \pi}-\frac{U}{2 \pi} \\
& B_{22}=\bar{v}-\frac{\lambda_{11}^{\rho}+\lambda_{22}^{\rho}-2 \lambda_{12}^{\rho}}{2 \pi}+\frac{C_{11}^{\rho}+C_{22}^{\rho}-2 C_{12}^{\rho}}{2 \pi}=\bar{v}+\frac{V_{2 k_{F 1}}}{4 \pi} \\
& +\frac{V_{2 k_{F 2}}}{4 \pi}-\frac{V_{\pi / 2}}{2 \pi}+\frac{V_{k_{F 1}-k_{F 2}}}{2 \pi}-\frac{U}{2 \pi} \\
& B_{12}=B_{21}=v_{r}-\frac{\lambda_{11}^{\rho}-\lambda_{22}^{\rho}}{2 \pi}+\frac{C_{11}^{\rho}-C_{22}^{\rho}}{2 \pi}=v_{r}+\frac{V_{2 k_{F 1}}}{4 \pi}-\frac{V_{2 k_{F 2}}}{4 \pi},
\end{aligned}
$$

where

$$
\bar{v} \equiv \frac{v_{1}+v_{2}}{2}, \quad v_{r} \equiv \frac{v_{1}-v_{2}}{2} .
$$

The couplings $\lambda_{a b}^{\rho}$ of the right-left mixing interactions $\mathcal{H}_{R L}^{\rho}$ enter with opposite signs in $\mathbf{A}$ and $\mathbf{B}$ and directly affect Luttinger parameters, while the couplings $C_{a b}^{\rho}$ of $\mathcal{H}_{\text {chiral }}^{\rho}$ enter with the same sign and give velocity renormalizations.

From the final expressions in terms of $V_{Q}$, we see that the $Q=0$ component enters only in $A_{11}$. This can be understood by considering the $Q=0$ part of the interaction, ${ }^{24}$

$$
\sum_{x, x^{\prime}} V\left(x-x^{\prime}\right) n(x) n\left(x^{\prime}\right) \rightarrow V_{Q=0} \int_{x}[\rho(x)]^{2},
$$

where $\rho(x)=2 \partial_{x} \theta_{\rho+} / \pi$ is the coarse-grained electron density.

Note also that the $-U /(2 \pi)$ in the diagonal matrix elements is due to our more careful treatment of the on-site repulsion, which we first write as $U n_{\uparrow}(x) n_{\downarrow}(x)$ and then bosonize. We obtain harmonic description of the C2S2 phase by combining the spin and charge sectors. The latter twomode system $\mathcal{L}^{\rho}$ has nontrivial Luttinger parameters, which can be determined from the matrices $\mathbf{A}$ and $\mathbf{B}$ (cf. Appendix). The fixed-point matrix elements will differ somewhat from the bare values above, but we ignore this in our crude analysis of the intermediate coupling regime.

To complete the bosonization of the four-fermion interactions, Eqs. (7)-(10), the $w_{12}^{\rho / \sigma}$ terms give ${ }^{14,27}$

$$
\begin{aligned}
W \equiv & \left(w_{12}^{\rho} J_{R 12} J_{L 12}-w_{12}^{\sigma} \vec{J}_{R 12} \cdot \vec{J}_{L 12}\right)+\text { H.c. } \\
= & \cos \left(2 \varphi_{\rho-}\right)\left\{4 w_{12}^{\rho}\left[\cos \left(2 \varphi_{\sigma-}\right)-\hat{\Gamma} \cos \left(2 \theta_{\sigma_{-}}\right)\right]\right. \\
& \left.-w_{12}^{\sigma}\left[\cos \left(2 \varphi_{\sigma_{-}}\right)+\hat{\Gamma} \cos \left(2 \theta_{\sigma_{-}}\right)+2 \hat{\Gamma} \cos \left(2 \theta_{\sigma_{+}}\right)\right]\right\},
\end{aligned}
$$

where $\hat{\Gamma}=\eta_{1 \uparrow} \eta_{1 \downarrow} \eta_{2 \uparrow} \eta_{2 \downarrow}$. We see that $W$ couples the charge and spin sectors. In the $\mathrm{C} 2 \mathrm{~S} 2$ theory described above, its scaling dimension is

$$
\Delta[W]=\Delta\left[\cos \left(2 \varphi_{\rho-}\right)\right]+1,
$$

where $\Delta\left[\cos \left(2 \varphi_{\rho-}\right)\right]$ is evaluated in the Lagrangian $\mathcal{L}^{\rho}$, while the contribution 1 comes from the spin sector. For the $\mathrm{C} 2 \mathrm{~S} 2$ theory to be consistent, the $W$ term must be irrelevant, $\Delta[W]>2$. Once the $W$ renormalizes to zero, the charge and spin sectors decouple. We thus have precise parallel with the weak coupling analysis of the C2S2 fixed point in Sec. II.

On the other hand, if $\Delta[W]<2$, the $W$ term becomes relevant and the $\mathrm{C} 2 \mathrm{~S} 2$ state is unstable. In this case, $\varphi_{\rho-}$ will get pinned and also the spin sector will become gapped. Only the " $\rho+"$ mode remains gapless and the system is some C1S0 conducting phase.

\section{B. Mott insulator driven by umklapp interaction: Intermediate coupling procedure out of the $\mathrm{C2S2}$}

The weak coupling analysis in Sec. II misses the possibility of gapping out the overall charge mode $\theta_{\rho+}$ since there are no four-fermion umklapp terms allowed in the two-band system. However, the half-filled electronic system does become a Mott insulator for sufficiently strong repulsion. In the theoretical description, this is achieved by an eight-fermion umklapp term, ${ }^{14}$

$$
\begin{aligned}
H_{8} & =v_{8}\left(c_{R 1 \uparrow}^{\dagger} c_{R 1 \downarrow}^{\dagger} c_{R 2 \uparrow}^{\dagger} c_{R 2 \downarrow}^{\dagger} c_{L 1 \uparrow} c_{L 1 \downarrow} c_{L 2 \uparrow} c_{L 2 \downarrow}+\text { H.c. }\right) \\
& =2 v_{8} \cos \left(4 \theta_{\rho+}\right) .
\end{aligned}
$$

At weak coupling, this term has scaling dimension $\Delta\left[H_{8}\right]$ $=4$ and is strongly irrelevant. However, from Eq. (46) we see that overall repulsive interaction stiffens the $\theta_{\rho+}$ mode and lowers the scaling dimension of $H_{8}$. For sufficiently strong repulsion, $\Delta\left[H_{8}\right]$ drops below 2 and the umklapp becomes 
relevant; $\theta_{\rho+}$ gets pinned and we obtain a Mott insulator.

Our intermediate coupling procedure is as follows. Using the harmonic theory of the $\mathrm{C} 2 \mathrm{~S} 2$ phase, we calculate the scaling dimensions $\Delta[W]$, Eq. (49), and $\Delta\left[H_{8}\right]$ $=\Delta\left[\cos \left(4 \theta_{\rho_{+}}\right)\right]$from the Lagrangian $\mathcal{L}^{\rho}$, Eq. (38). Details are described in Appendix and calculations are done numerically in the end.

If both $\Delta[W]$ and $\Delta\left[H_{8}\right]$ are larger than 2, the C2S2 metal is stable. As interactions increase, eventually either $W$ or $H_{8}$ becomes relevant. In general, there are two cases.

(1) If $H_{8}$ becomes relevant first, we pin $\theta_{\rho+}$ and enter C1S2 Mott insulator. To be more precise, we can further qualify the label as " $\mathrm{C} 1[\rho-] \mathrm{S} 2$ "; the remaining "charge" mode " $\rho-$ " represents local current loop fluctuations and does not conduct. This is the spin liquid phase called spin Bose-metal in Ref. 14 and described in detail there. Exploring conditions for finding such phase in electronic models is our main goal here.

(2) On the other hand, if the $W$ term becomes relevant first, we enter C1S0 conducting state with a spin gap (more precisely, "C1 $[\rho+] \mathrm{S} 0 ")$.

Some reservations are in order. First, we use bare values of the couplings in the $\mathbf{A}$ and $\mathbf{B}$ matrices, which is not accurate since the couplings experience initial flows, cf. Sec. III B Second, we consider only instabilities driven by changes in the harmonic $\mathcal{L}^{\rho}$ theory as they translate to scaling dimensions of the $H_{8}$ and $W$ terms, i.e., we effectively treat the latter as small. We also assume that the spin sector is near the fixed point with all $\lambda_{a b}^{\sigma}$ marginally irrelevant and small. We will address these reservations after presenting results of the above procedure. Keeping these remarks in mind, we now describe how we analyze phases out of the $\mathrm{C} 1[\rho-] \mathrm{S} 2$ and $\mathrm{C} 1[\rho+] \mathrm{S} 0$ in the same procedure.

\section{Instability out of $\mathrm{C} 1[\rho-] \mathrm{S} 2$ driven by spin-charge coupling $W$}

In the present analysis focusing on the $\rho+$ and $\rho$ - fields, we can also crudely estimate the extent of the C1S2 or C1S0 phases once either happens out of the C2S2. Suppose the umklapp $H_{8}$ is relevant first and we are in the C1S2 phase. We still need to remember the $W$ term since it can become relevant if we continue increasing the interaction strength. To estimate the scaling dimension of the $W$ term, we assume now that the $\theta_{\rho+}$ field is massive and integrate out $\theta_{\rho+}$ and $\varphi_{\rho+}$. Mathematically this amounts to sending $A_{11} \rightarrow \infty$, and we obtain

$$
\Delta\left[W ; \theta_{\rho+} \text { is pinned }\right]=\left[\frac{A_{22} B_{11}}{B_{11} B_{22}-B_{12}^{2}}\right]^{1 / 2}+1 .
$$

This assumption is approximate but reasonable, since once the parameters are such that the system is in the C1S2 phase, the relevant $H_{8}$ will grow and quickly stiffen the $A_{11}$ in positive feedback loop.

The $\mathrm{C} 1 \mathrm{~S} 2$ phase is stable if $\Delta[W]>2$, and this analysis is similar to the stability analysis of the SBM in Ref. 14. If $\Delta[W]$ drops below 2 , the $W$ term becomes relevant and the $\varphi_{\rho-}$ field will be pinned, together with gapping out the spin sector, cf. Eq. (48). The final result is some "COSO" phase, whose precise character depends on the details of the cou-

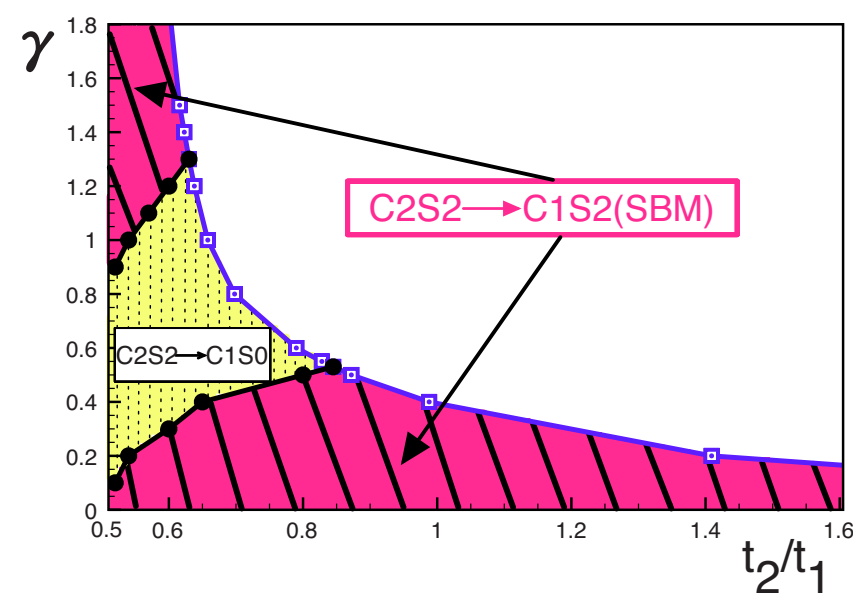

FIG. 6. (Color online) Projection of phases obtained out of the $\mathrm{C} 2 \mathrm{~S} 2$ of Fig. 4 as we increase overall repulsion strength $V$, which we imagine to be the $z$ axis perpendicular to the page (Fig. 7 gives one cut at $\gamma=0.4$ with such $V$ axis shown explicitly). The results are obtained in the intermediate coupling procedure as explained in the text. White region is $\mathrm{C} 1 \mathrm{~S} 0$ at weak coupling, cf. Fig. 4, and is not considered here.

plings $w_{12}^{\rho / \sigma}$. This is studied in Sec. IVB of Ref. 14. For the present repulsive electron model, we have $w_{12}^{\rho}, w_{12}^{\sigma}>0$, so the resulting COSO is likely a period-2 valence bond solid (VBS). ${ }^{14}$ This connects to dimerized phase in the $J_{1}-J_{2}$ spin chain appropriate in the strong interaction limit of the electron system.

\section{Instability out of $\mathrm{C} 1[\rho+] \mathrm{S} 0$ driven by umklapp $\mathrm{H}_{8}$}

Suppose now the $W$ interaction becomes relevant first. From Eq. (48), it is natural that $\varphi_{\rho-}$ is pinned, the spin sector gets gapped, and we are in C1S0 phase. Here we postulate mass for $\varphi_{\rho-}$ (essentially sending $B_{22} \rightarrow \infty$ ) and calculate the effective scaling dimension of the umklapp term,

$$
\Delta\left[H_{8} ; \varphi_{\rho-} \text { is pinned }\right]=4\left[\frac{B_{11} A_{22}}{A_{11} A_{22}-A_{12}^{2}}\right]^{1 / 2} .
$$

If $\Delta\left[H_{8}\right]>2$, the $\mathrm{C} 1 \mathrm{~S} 0$ is stable. Once $\Delta\left[H_{8}\right]$ drops below 2 , the overall charge mode $\theta_{\rho+}$ is pinned and we obtain fully gapped Mott insulator COSO, which is likely the same period-2 VBS discussed earlier.

\section{Numerical results}

We consider the same models with extended densitydensity interactions as in the weak coupling analysis in Sec. II E, parking ourselves initially in the C2S2 phase in Figs. 4 and 5. From the preceding discussion, we can obtain two phases out of the $\mathrm{C} 2 \mathrm{~S} 2$ upon increasing interaction strength-either $\mathrm{C} 1[\rho+] \mathrm{S} 0$ or $\mathrm{C} 1[\rho-] \mathrm{S} 2$. To visualize the results, we imagine adding the overall interaction strength $V$ as the $z$-axis to Figs. 4 and 5. We then project down which phase happens first for each such vertical line out of C2S2. Calculations are done numerically and the results are shown in Figs. 6 and 9. In Fig. 7 we take a cut through Fig. 6 at $\gamma=0.4$ and show details of the phase diagram in the $t_{2} / t_{1}$ $-V$ plane. 


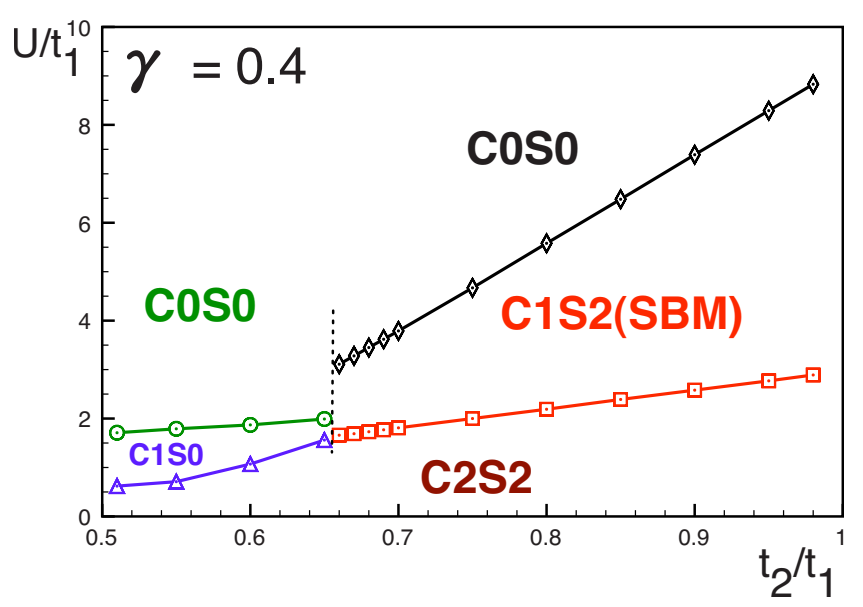

FIG. 7. (Color online) Intermediate coupling analysis of the model with potential Eq. (32) for $\kappa=0.5$ and $\gamma=0.4$. Here the horizontal range is equal to the extent of the $\mathrm{C} 2 \mathrm{~S} 2$ phase in the weak coupling analysis from Fig. 4 . We start in the $\mathrm{C} 2 \mathrm{~S} 2$ at small $U$. The boundary where the charge-spin coupling term $W$ becomes relevant first is indicated with blue triangles and the system goes into the $\mathrm{C} 1 \mathrm{~S} 0$; the next stage where the C1S0 in turn becomes unstable and the system goes into the COSO is marked with green circles. The boundary where the umklapp term $H_{8}$ becomes relevant first is indicated with red squares and the system goes into the C1S2, which is the SBM phase of Ref. 14; upon further increase in the interaction strength the C1S2 eventually becomes unstable and goes to the $\mathrm{COSO}$ at locations marked with black diamonds. Note that the discontinuity shown with dotted vertical line is not meaningful and is due to our crude analysis performed separately out of the C1S0 and $\mathrm{C} 1 \mathrm{~S} 2$; in either case, the final C0S0 is likely the same phase. Also note that the $\mathrm{C} 1$ mode content is distinct in the $\mathrm{C} 1[\rho+] \mathrm{S} 0$ (conducting) and $\mathrm{C} 1[\rho-] \mathrm{S} 2$ (insulating) cases and any transition between them is first order. The $\mathrm{C} 2 \mathrm{~S} 2$ to $\mathrm{C} 1 \mathrm{~S} 2$ transition is Kosterlitz-Thouless-like.

\section{Intermediate coupling phase diagram for model with potential [Eq. (32)]}

Figure 6 shows results for the model potential Eq. (32). We can see that in two regimes $\gamma \geq 1.2$ and $\gamma \leq 0.4$ we exit from the $\mathrm{C} 2 \mathrm{~S} 2$ into the $\mathrm{C} 1 \mathrm{~S} 2$. The two limits can be understood analytically.

In the large $\gamma$ case, we can replace all $V_{Q}$ by simply $U$. The matrices $\mathbf{A}$ and $\mathbf{B}$ defined in Eq. (39)-(44) become

$$
\mathbf{A}=\left(\begin{array}{cc}
\bar{v}+\frac{2 U}{\pi} & v_{r} \\
v_{r} & \bar{v}
\end{array}\right), \quad \mathbf{B}=\left(\begin{array}{cc}
\bar{v} & v_{r} \\
v_{r} & \bar{v}
\end{array}\right) .
$$

We see that $U$ only contributes to $A_{11}$. This monotonically "stiffens" the $\theta_{\rho+}$ (lowering $\Delta\left[H_{8}\right]$ ) but "softens" the $\varphi_{\rho-}$ (increasing $\Delta[W]$ ). Therefore we only expect the C1S2 phase out of the $\mathrm{C} 2 \mathrm{~S} 2$ as found in the numerical calculations.

On the other hand, for small $\gamma$ we can see from Eq. (33) that $V_{Q=0}$ will dominate over $V_{Q \neq 0}$. Keeping only $V_{Q=0}$, the matrices $\mathbf{A}$ and $\mathbf{B}$ become

$$
\mathbf{A} \simeq\left(\begin{array}{cc}
\bar{v}+\frac{4 V_{Q=0}}{\pi} & v_{r} \\
v_{r} & \bar{v}
\end{array}\right), \quad \mathbf{B} \simeq\left(\begin{array}{cc}
\bar{v} & v_{r} \\
v_{r} & \bar{v}
\end{array}\right)
$$

Thus the small $\gamma$ case has similar mathematical structure to the large $\gamma$ case. The physical difference is that here the transition to the $\mathrm{C} 1 \mathrm{~S} 2$ is driven by the $V_{Q=0}$ instead of the on-site Hubbard $U$. Note also that since $V_{Q=0} \simeq 2 \kappa U / \gamma$ for $\gamma \ll 1$, the transition requires only small values of $U$, which is why we can ignore all $V_{Q \neq 0}$ compared to the band velocities.

Now we consider a cut at $\gamma=0.4$ to see more details in the $t_{2} / t_{1}-V$ plane. The results are shown in Fig. 7. Compared with the two limits $\gamma \gg 1$ and $\gamma \ll 1$ above, all possibilities that we discussed out of the $\mathrm{C} 2 \mathrm{~S} 2$ are realized here. The C1S0 phase appears for $t_{2} / t_{1}<0.65$ for some quantitative reasons. Various $V_{Q}$ are all of the same order, unlike the $\gamma$ $\ll 1$ case. At the same time, they have some nontrivial $Q$-dependence, unlike the $\gamma \gg 1$ case, which is somehow enough to make the $W$ term become relevant and preempt the umklapp term. Note that for small interactions the scaling dimension of the $W$ term can be obtained from the weak coupling RG equations for the $w_{12}^{\rho / \sigma}$ in Sec. II B by setting all $\lambda_{a b}^{\sigma}=0$ (since we ignore the spin sector in the present procedure). Thus, $\Delta[W]=2+\Lambda^{\rho}$, where $\Lambda^{\rho}$ is defined in Eq. (31). Since $\Lambda^{\rho}$ can only decrease under the weak coupling RG and the shaded $\mathrm{C} 2 \mathrm{~S} 2$ region in Fig. 4 was found to be stable, we expect $\Delta[W]$ here to increase with $V$ for small $V$, in agreement with numerical calculations. However, we find that $\Delta[W]$ eventually starts to decrease with increasing $V$ and can become relevant before the umklapp. This is a quantitative matter and comes from putting together all interactions $\mathcal{H}_{R L}^{\rho}$ and $\mathcal{H}_{\text {chiral }}^{\rho}$, Eqs. (7)-(9), in the intermediate coupling procedure. Such numerical calculations give us that the C2S2 can exit into the C1S0 phase. For larger $t_{2} / t_{1}>0.65$ in Fig. 7 , we obtain the sought for C1S2 spin liquid phase.

This concludes the presentation of formal results within the particular procedure for intermediate scale analysis. Let us now think how to combine the weak and intermediate coupling approaches more realistically and see where our results are more robust.

First of all, in the weak coupling analysis the C2S2 phase is unstable beyond the shaded regions in Figs. 4 and 5. However, this is lost in the specific intermediate coupling procedure, which, when applied for small coupling, would give C2S2 essentially everywhere. For example, in Fig. 7 we see monotonic growth of the $\mathrm{C} 2 \mathrm{~S} 2$ phase with $t_{2} / t_{1}$ past the point where the weak coupling analysis predicts instability. The reason for this discrepancy is the complete neglect of the spin sector in the formal intermediate scale procedure. Indeed, in the weak coupling analysis, the instabilities manifest dramatically once one of the $\lambda_{a a}^{\sigma}$ becomes negative, causing runaway flows. This can happen even when the bare $\lambda_{a a}^{\sigma}$ are repulsive because they are renormalized downwards and can be driven negative by the $w_{12}^{\rho / \sigma}$ contributions in Eqs. (26) and (27), where we assume $w_{12}^{\rho} w_{12}^{\sigma}>0$. Also, the $\lambda^{\sigma}$ couplings feed back into the flow of $w_{12}^{\rho / \sigma}$, so the RG flow behavior is even more complex. So far we have dealt with this inadequacy of the intermediate scale procedure by simply cutting 


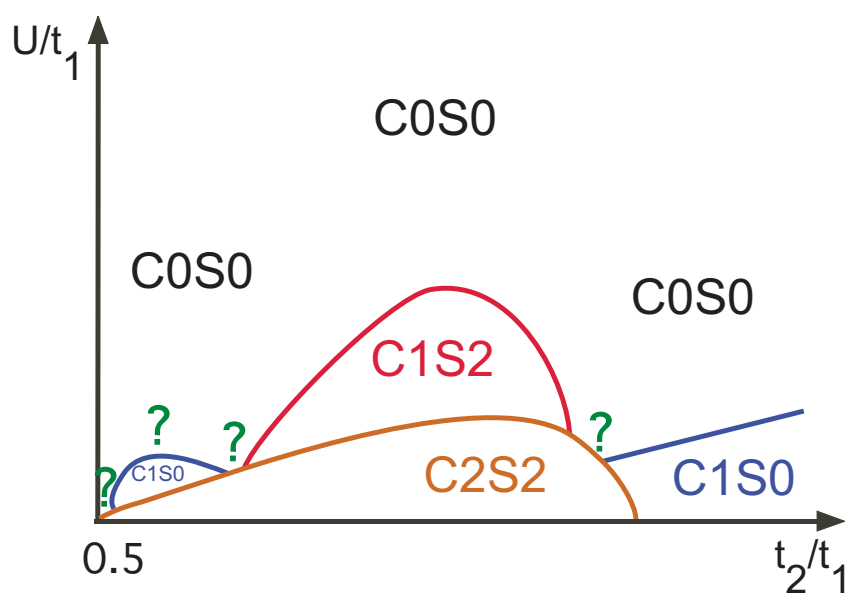

FIG. 8. (Color online) Schematic merging of the weak and intermediate coupling results in the model regimes like in Fig. 7 in the whole range with $t_{2} / t_{1}>0.5$. In weak coupling, the C2S2 phase is unstable beyond the shaded region in Fig. 4. However, due to the crudeness of our intermediate coupling procedure, Fig. 7 shows monotonic growth of the $\mathrm{C} 2 \mathrm{~S} 2$ phase with $t_{2} / t_{1}$ past this instability. This discrepancy arises because our intermediate coupling procedure completely ignores the spin sector. More realistically, we expect the $\mathrm{C} 2 \mathrm{~S} 2$ phase to peak somewhere in the middle of the range shown in Fig. 7 and be bounded by the C1S0 for larger $t_{2} / t_{1}$. Similar considerations apply to the $\mathrm{C} 1 \mathrm{~S} 2$ phase, which is bounded by the COSO.

it at the $\mathrm{C} 2 \mathrm{~S} 2$ boundaries determined from the weak coupling analysis. More realistically, we expect the extent of the $\mathrm{C} 2 \mathrm{~S} 2$ phase to peak somewhere in the middle of the range shown in Fig. 7 and decrease toward the right boundary. Similar considerations apply to the C1S2 phase, which is likely confined within the same $t_{2} / t_{1}$ range as the C2S2. Therefore, the $t_{2} / t_{1}-U / t_{1}$ phase diagram should be more like Fig. 8.

We can also discuss our earlier reservation about using bare values of the couplings instead of some renormalized values. Thinking about some RG treatment, we expect that crude patterns of how various couplings affect each other are likely similar at intermediate and weak couplings. Now if we formally take the flow equations from Sec. II B, the outcome does not depend on the initial interaction scale, so we would conclude the $\mathrm{C} 2 \mathrm{~S} 2$ phase throughout the shaded region in Figs. 4. The weak coupling flow equations miss velocity renormalizations due to chiral interactions, but these are not expected to flow strongly and are treated reasonably in the intermediate coupling analysis. The fact that the couplings are now finite and comparable with bare band energies is also treated reasonably at intermediate coupling due to the power of bosonization, so the outlined forging of weak and intermediate scales seems appropriate. Finally, the umklapp term that is missing in the weak coupling approach will feed into stiffening of $\theta_{\rho+}$ only, which is good for the first instability out of the $\mathrm{C} 2 \mathrm{~S} 2$ to be into the C1S2 spin liquid.

We think that our conclusions are more robust for small $\gamma$ where the extent of the C2S2 phase is larger and also the longer-ranged potential is feeding precisely into stiffening the overall charge field $\theta_{\rho+}$, which is good for going to the

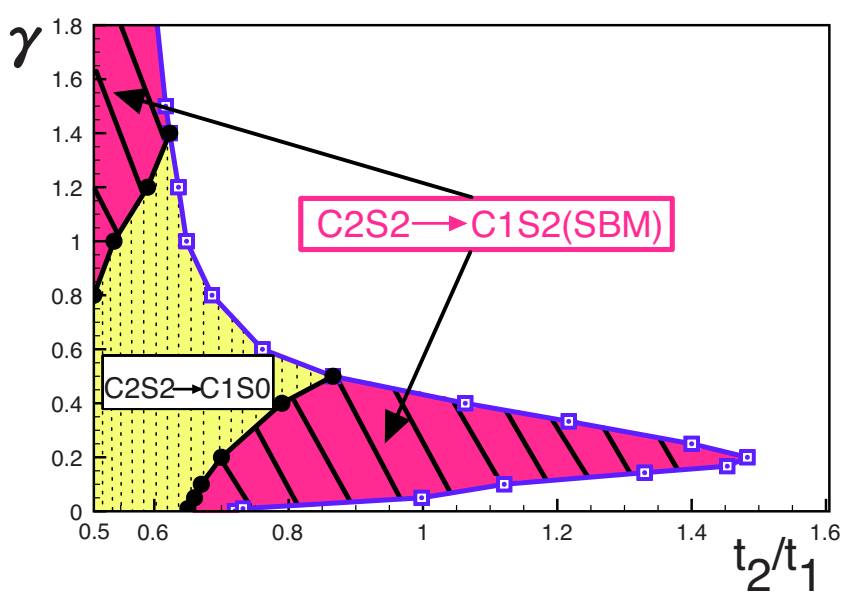

FIG. 9. (Color online) Same as Fig. 6 but for the model with interactions [Eq. (32)] truncated at the fourth neighbor and starting out of the C2S2 of Fig. 5.

C1S2 phase. On the other hand, results at medium to large $\gamma$ are likely less reliable, with different scenarios depending on quantitative issues.

\section{Intermediate coupling phase diagram for model with potential [Eq. (32)] truncated at the fourth neighbor}

Figure 9 shows results of the intermediate coupling analysis for the model with interactions truncated at the fourth neighbor, cf. Sec. II E 2 . We have a rather similar story to Fig. 6, except that the initial $\mathrm{C} 2 \mathrm{~S} 2$ region is bounded. Large part of the $\mathrm{C} 2 \mathrm{~S} 2$ phase exits into the $\mathrm{C} 1 \mathrm{~S} 2$ spin liquid upon increasing interactions, and our results are probably more robust near $\gamma \sim 0.2-0.3$ where the $\mathrm{C} 2 \mathrm{~S} 2$ has the largest extent along the $t_{2} / t_{1}$ axis.

\section{SUMMARY AND DISCUSSION}

To summarize, in this paper we consider electronic models for realizing spin Bose-metal (spin liquid) phase on the two-leg triangular strip found in Ref. 14 in spin-1/2 model with ring exchanges. We identify the SBM with the C1S2 Mott insulator of electrons.

In Sec. II, we start with a two-band electron system, which is C2S2. Instead of considering only the on-site Hubbard-type repulsion, ${ }^{18,20-22,26,28,29}$ we study generally longer-ranged density-density repulsion. This is motivated in part by the expectation that real Coulomb interaction is not screened in Mott insulator materials, so further neighbor repulsion can be significant, as brought up by recent $a b$ initio work $^{25}$ for the spin liquid material $\kappa-(\mathrm{ET})_{2} \mathrm{Cu}_{2}(\mathrm{CN})_{3}$. Using weak coupling $\mathrm{RG}$ analysis for the zigzag chain problem, ${ }^{17,18,23,27}$ we find that such extended interactions open much wider window of the $\mathrm{C} 2 \mathrm{~S} 2$ metal compared with the Hubbard model. The main results are shown in Figs. 4 and 5. In the first figure, we have essentially an independent control over the $Q=0$ part of the potential by allowing it to extend to far neighbors, and we identify the dominance of $V_{Q=0}$ as the main stabilizing force for the metal. In the second figure, we truncate interactions at the fourth neighbor to 
check the robustness of our conclusions, in view that such models may be easier to explore using numerical DMRG. Our detailed quasi-1D considerations agree with the intuition that in real metals electronic pairing instabilities are suppressed by the long-ranged piece of the Coulomb interaction. Such widening of the $\mathrm{C} 2 \mathrm{~S} 2$ region by extending the model interaction range is warranted if we want to bring the electronic ladder system closer to realistic situations in the $2 \mathrm{D}$ candidate spin liquid materials.

In Sec. III, we begin with stable C2S2 metal at weak coupling and use bosonization to extend the analysis to intermediate coupling by gradually increasing the overall repulsion strength. Within effective bosonic theory, we identify potential instabilities of the $\mathrm{C} 2 \mathrm{~S} 2$ phase to spin-charge interaction $W$ [Eq. (48)] and umklapp interaction $H_{8}$ [Eq. (50)]. The $W$ can drive the system into $\mathrm{C} 1[\rho+] \mathrm{S} 0$ phase with spin gap but still conducting along the chain, while the umklapp $H_{8}$ can produce $\mathrm{C} 1[\rho-] \mathrm{S} 2$ Mott insulator with three gapless modes, which is the desired SBM phase. We calculate the scaling dimensions of the $W$ and $H_{8}$ terms in the harmonic theory of the $\mathrm{C} 2 \mathrm{~S} 2$ metal using bare couplings in the charge sector and assuming stability in the spin sector-this constitutes our naive intermediate coupling procedure. The calculation of scaling dimensions is described in Appendix and is done numerically in the end.

We consider two cases depending on which of the terms $W$ or $H_{8}$ becomes relevant first and apply similar intermediate coupling approach inside the resulting phase. Assuming strong field pinning by the already relevant term, we calculate the scaling dimension of the remaining term and estimate when it eventually drives the system into fully gapped COSO paramagnet (which is likely connected to the dimerized phase of the $J_{1}-J_{2}$ Heisenberg model at strong coupling). With the help of such admittedly crude analysis, we can map out the phase diagram in weak to intermediate coupling regime as illustrated in schematic Fig. 8 (based on more naive Fig. 7). Figures 6 and 9 summarize our results and show where the C2S2 metal goes to the C1S2 (SBM spin liquid) upon increasing overall repulsion strength. We conclude that the C1S2 phase is quite natural out of the wider C2S2 metallic region, in particular when driven by extended repulsive interactions. It would be very interesting to confront our theoretical predictions with numerical DMRG studies of such electronic models with extended repulsion.

So far, we have approached the intermediate coupling Mott insulator from the weak coupling metallic side. One could try to attack the same problem starting from the strong coupling limit deep in the Mott insulator where Heisenberg spin-1/2 model is appropriate. As one nears the metallic phase, it becomes important to include multiple spin exchanges in the effective spin Hamiltonian to better capture charge fluctuations in the underlying electron system. ${ }^{7,15,16,30}$ This is the motivation behind Ref. 14 studying $J_{1}-J_{2}$ chain with additional four-spin ring exchanges. The concept study Ref. 14 allowed arbitrary variation in the ring coupling compared with the Heisenberg couplings. However, coming from an electronic model these do not vary independently and more exchange terms are also generated. It would be interesting to pursue such approach systematically studying effective spin models with multispin exchanges for realistic elec- tronic models to see if they harbor the SBM phase. We do not make such attempts here, but only give few simple observations on how the derivation of the spin model is modified in the presence of extended repulsion.

First of all, for the two spin exchanges, the familiar Hubbard model expression $J_{r r^{\prime}}=4 t_{r r^{\prime}}^{2} / U$ is modified to $J_{r r^{\prime}}$ $=4 t_{r r^{\prime}}^{2} /\left(V_{0}-V_{r-r^{\prime}}\right)$. The energy denominator is not simply the on-site $U=V_{0}$ but also includes interaction potential between the two sites $r$ and $r^{\prime}$. For example, Ref. 25 estimates $V_{1} / V_{0} \approx 0.43$ for the $\kappa$ - $(\mathrm{ET})_{2} \mathrm{Cu}_{2}(\mathrm{CN})_{3}$ spin liquid material, and this would significantly affect values of the exchange constants. Energy denominators for all virtual processes are similarly affected and take a form of a charging energy for the deviations from the background. Multispin exchange amplitudes are given by a product of electron tunneling amplitudes for a given virtual path divided by a product of such charging energies in intermediate states along the path. Thus, the multispin exchanges may in fact be relatively more important in systems with extended interactions.

As an extreme example, imagine a very slow decrease in $V\left(r-r^{\prime}\right)$ up to some distance $R$ (and perhaps a faster drop thereafter). Then all exchange loops up to such radius $R$ will have large amplitudes. The multispin exchanges encode the underlying kinetic energy of electrons, and our intuition is that this would like to retain some itinerancy in the spin degrees of freedom even when the charges are localized. From such strong to intermediate coupling perspective, it appears that extended interactions would tend to stabilize the SBM spin liquid near the insulator-metal transition, similar to our conclusion from the weak to intermediate coupling study in the quasi-1D models in this paper. It would be interesting to pursue such considerations more carefully and in realistic electronic models. We hope that our work will further stimulate numerical studies of such models on ladders and in two dimensions.

\section{ACKNOWLEDGMENTS}

We would like to thank M. P. A. Fisher, I. Gonzalez, R. Melko, and D. N. Sheng for useful discussions and M. P. A. Fisher for stimulating this work and critical reading of the manuscript. This research is supported by the A. P. Sloan Foundation and the National Science Foundation through Grant No. DMR-0907145.

\section{APPENDIX: DERIVATION OF $\Delta\left[\cos \left(4 \theta_{\rho+}\right)\right]$ AND $\Delta\left[\cos \left(2 \varphi_{\rho_{-}}\right)\right]$IN C2S2 PHASE}

Equation (38) gives quadratic Lagrangian for the charge sector. First, we redefine the fields which still satisfy the same commutation relations,

$$
\boldsymbol{\Theta}=\mathbf{S} \cdot \boldsymbol{\Theta}_{1}, \quad \boldsymbol{\Phi}=\mathbf{S} \cdot \boldsymbol{\Phi}_{1} .
$$

Here $\mathbf{S}$ is an orthogonal $2 \times 2$ matrix diagonalizing the matrix A,

$$
\mathbf{S}^{T} \cdot \mathbf{A} \cdot \mathbf{S}=\left(\begin{array}{cc}
A_{1} & 0 \\
0 & A_{2}
\end{array}\right) \equiv \mathbf{A}_{D} .
$$

The Lagrangian becomes 


$$
\begin{aligned}
\mathcal{L}^{\rho}= & \frac{1}{2 \pi}\left[\partial_{x} \boldsymbol{\Theta}_{1}^{T} \cdot \mathbf{A}_{D} \cdot \partial_{x} \boldsymbol{\Theta}_{1}+\partial_{x} \boldsymbol{\Phi}_{1}^{T} \cdot \mathbf{S}^{T} \cdot \mathbf{B} \cdot \mathbf{S} \cdot \partial_{x} \boldsymbol{\Phi}_{1}\right] \\
& +\frac{i}{\pi} \partial_{x} \boldsymbol{\Theta}_{1}^{T} \cdot \partial_{\tau} \boldsymbol{\Phi}_{1}
\end{aligned}
$$

Define another set of conjugate fields,

$$
\boldsymbol{\Theta}_{1}=\frac{1}{\sqrt{\mathbf{A}_{D}}} \cdot \boldsymbol{\Theta}_{2}, \quad \boldsymbol{\Phi}_{1}=\sqrt{\mathbf{A}_{D}} \cdot \boldsymbol{\Phi}_{2} .
$$

We obtain

$$
\mathcal{L}^{\rho}=\frac{1}{2 \pi}\left[\partial_{x} \boldsymbol{\Theta}_{2}^{T} \cdot \partial_{x} \boldsymbol{\Theta}_{2}+\partial_{x} \boldsymbol{\Phi}_{2}^{T} \cdot \mathbf{B}^{\prime} \cdot \partial_{x} \boldsymbol{\Phi}_{2}\right]+\frac{i}{\pi} \partial_{x} \boldsymbol{\Theta}_{2}^{T} \cdot \partial_{\tau} \boldsymbol{\Phi}_{2}
$$

where

$$
\mathbf{B}^{\prime} \equiv \sqrt{\mathbf{A}_{D}} \cdot \mathbf{S}^{T} \cdot \mathbf{B} \cdot \mathbf{S} \cdot \sqrt{\mathbf{A}_{D}}
$$

We use the same trick to diagonalize matrix $\mathbf{B}^{\prime}$,

$$
\boldsymbol{\Theta}_{2}=\mathbf{R} \cdot \boldsymbol{\Theta}_{3}, \quad \boldsymbol{\Phi}_{2}=\mathbf{R} \cdot \boldsymbol{\Phi}_{3},
$$

where $\mathbf{R}$ is an orthogonal matrix which satisfies,

$$
\mathbf{R}^{T} \cdot \mathbf{B}^{\prime} \cdot \mathbf{R}=\left(\begin{array}{cc}
B_{1}^{\prime} & 0 \\
0 & B_{2}^{\prime}
\end{array}\right) \equiv \mathbf{B}_{D}^{\prime}
$$

The Lagrangian becomes,

$$
\mathcal{L}^{\rho}=\frac{1}{2 \pi}\left[\partial_{x} \boldsymbol{\Theta}_{3}^{T} \cdot \partial_{x} \boldsymbol{\Theta}_{3}+\partial_{x} \boldsymbol{\Phi}_{3}^{T} \cdot \mathbf{B}_{D}^{\prime} \cdot \partial_{x} \boldsymbol{\Phi}_{3}\right]+\frac{i}{\pi} \partial_{x} \boldsymbol{\Theta}_{3}^{T} \cdot \partial_{\tau} \boldsymbol{\Phi}_{3} .
$$

Now we can calculate the scaling dimension of $\cos \left(4 \theta_{\rho+}\right)$ and $\cos \left(2 \varphi_{\rho_{-}}\right)$from Eq. (A9) through relations

$$
\begin{gathered}
\boldsymbol{\Theta}=\mathbf{S} \cdot \frac{1}{\sqrt{\mathbf{A}_{D}}} \cdot \mathbf{R} \cdot \boldsymbol{\Theta}_{3}, \\
\boldsymbol{\Phi}=\mathbf{S} \cdot \sqrt{\mathbf{A}_{D}} \cdot \mathbf{R} \cdot \boldsymbol{\Phi}_{3},
\end{gathered}
$$

and scaling dimensions of the final fields,

$$
\Delta\left[e^{i \Theta_{3}}\right]=\frac{\sqrt{\mathbf{B}_{D}^{\prime}}}{4}, \quad \Delta\left[e^{i \boldsymbol{\Phi}_{3}}\right]=\frac{1}{4 \sqrt{\mathbf{B}_{D}^{\prime}}},
$$

where the right hand sides mean corresponding diagonal matrix elements. Therefore, we find general form for the dimensions we are interested in

$$
\begin{aligned}
\Delta\left[\cos \left(4 \theta_{\rho+}\right)\right]= & 4 \sqrt{B_{1}^{\prime}}\left(\frac{S_{11} R_{11}}{\sqrt{A_{1}}}+\frac{S_{12} R_{21}}{\sqrt{A_{2}}}\right)^{2} \\
& +4 \sqrt{B_{2}^{\prime}}\left(\frac{S_{11} R_{12}}{\sqrt{A_{1}}}+\frac{S_{12} R_{22}}{\sqrt{A_{2}}}\right)^{2}
\end{aligned}
$$

$$
\begin{aligned}
\Delta\left[\cos \left(2 \varphi_{\rho-}\right)\right]= & \frac{\left(\sqrt{A_{1}} S_{21} R_{11}+\sqrt{A_{2}} S_{22} R_{21}\right)^{2}}{\sqrt{B_{1}^{\prime}}} \\
& +\frac{\left(\sqrt{A_{1}} S_{21} R_{12}+\sqrt{A_{2}} S_{22} R_{22}\right)^{2}}{\sqrt{B_{2}^{\prime}}},
\end{aligned}
$$

where $S_{a b}$ and $R_{a b}$ are matrix elements of $\mathbf{S}$ and $\mathbf{R}$.
${ }^{1}$ Y. Shimizu, K. Miyagawa, K. Kanoda, M. Maesato, and G. Saito, Phys. Rev. Lett. 91, 107001 (2003).

${ }^{2}$ Y. Kurosaki, Y. Shimizu, K. Miyagawa, K. Kanoda, and G. Saito, Phys. Rev. Lett. 95, 177001 (2005).

${ }^{3}$ S. Yamashita, Y. Nakazawa, M. Oguni, Y. Oshima, H. Nojiri, Y. Shimizu, K. Miyagawa, and K. Kanoda, Nat. Phys. 4, 459 (2008).

${ }^{4}$ M. Yamashita, N. Nakata, Y. Kasahara, T. Sasaki, N. Yoneyama, N. Kobayashi, S. Fujimoto, T. Shibauchi, and Y. Matsuda, Nat. Phys. 5, 44 (2009).

${ }^{5}$ T. Itou, A. Oyamada, S. Maegawa, M. Tamura, and R. Kato, Phys. Rev. B 77, 104413 (2008).

${ }^{6}$ Y. Okamoto, M. Nohara, H. Aruga-Katori, and H. Takagi, Phys. Rev. Lett. 99, 137207 (2007).

${ }^{7}$ O. I. Motrunich, Phys. Rev. B 72, 045105 (2005).

${ }^{8}$ S.-S. Lee and P. A. Lee, Phys. Rev. Lett. 95, 036403 (2005).

${ }^{9}$ Y. Zhou, P. A. Lee, T.-K. Ng, and F.-C. Zhang, Phys. Rev. Lett. 101, 197201 (2008).

${ }^{10}$ M. J. Lawler, A. Paramekanti, Y. B. Kim, and L. Balents, Phys. Rev. Lett. 101, 197202 (2008).

${ }^{11}$ T. Senthil, Phys. Rev. B 78, 045109 (2008).
${ }^{12}$ D. Podolsky, A. Paramekanti, Y. B. Kim, and T. Senthil, Phys. Rev. Lett. 102, 186401 (2009).

${ }^{13}$ P. A. Lee, N. Nagaosa, and X.-G. Wen, Rev. Mod. Phys. 78, 17 (2006).

${ }^{14}$ D. N. Sheng, O. I. Motrunich, and M. P. A. Fisher, Phys. Rev. B 79, 205112 (2009).

${ }^{15}$ A. D. Klironomos, J. S. Meyer, T. Hikihara, and K. A. Matveev, Phys. Rev. B 76, 075302 (2007).

${ }^{16}$ J. S. Meyer and K. A. Matveev, J. Phys.: Condens. Matter 21, 023203 (2009).

${ }^{17}$ M. Fabrizio, Phys. Rev. B 54, 10054 (1996).

${ }^{18}$ K. Louis, J. V. Alvarez, and C. Gros, Phys. Rev. B 64, 113106 (2001).

${ }^{19}$ C. Gros, K. Hamacher, and W. Wenzel, EPL 69, 616 (2005).

${ }^{20}$ S. Daul and R. M. Noack, Phys. Rev. B 61, 1646 (2000).

${ }^{21}$ R. Arita, K. Kuroki, H. Aoki, and M. Fabrizio, Phys. Rev. B 57, 10324 (1998).

${ }^{22}$ G. I. Japaridze, R. M. Noack, D. Baeriswyl, and L. Tincani, Phys. Rev. B 76, 115118 (2007).

${ }^{23}$ L. Balents and M. P. A. Fisher, Phys. Rev. B 53, 12133 (1996).

${ }^{24}$ C. Kane, L. Balents, and M. P. A. Fisher, Phys. Rev. Lett. 79, 5086 (1997). 
${ }^{25}$ K. Nakamura, Y. Yoshimoto, T. Kosugi, R. Arita, and M. Imada, J. Phys. Soc. Jpn. 78, 083710 (2009).

${ }^{26}$ H. C. Kandpal, I. Opahle, Y.-Z. Zhang, H. O. Jeschke, and R. Valenti, Phys. Rev. Lett. 103, 067004 (2009).

${ }^{27}$ H.-H. Lin, L. Balents, and M. P. A. Fisher, Phys. Rev. B 56, 6569 (1997)
${ }^{28}$ M. Capello, F. Becca, M. Fabrizio, S. Sorella, and E. Tosatti, Phys. Rev. Lett. 94, 026406 (2005).

${ }^{29}$ L. F. Tocchio, A. Parola, C. Gros, and F. Becca, Phys. Rev. B 80, 064419 (2009).

${ }^{30}$ A. H. MacDonald, S. M. Girvin, and D. Yoshioka, Phys. Rev. B 37, 9753 (1988). 